\title{
Testing of Techniques for Improvement of Conductivity of Electrically Conductive Adhesives
}

\author{
Pavel Mach ${ }^{1}$, Lukáš Richter ${ }^{1}$, Alena Pietriková ${ }^{2}$ \\ ${ }^{1}$ Czech Technical University in Prague, Faculty of Electrical Engineering \\ Technicka 2, 16627 Prague 6, Czech Republic \\ Email:mach@fel.cvut.cz, Phone: ++420 22435 2214, Fax: ++420 224363949 \\ ${ }^{2}$ Technical University of Kosice, Faculty of Electrical Engineering and Informatics \\ Letna 9, 04200 Kosice, Slovak Republic \\ Email: Alena.Pietrikova@tuke.sk, Phone: ++421 55 6023194, Fax: ++421 556023195
}

\begin{abstract}
The paper presents methods, which have been used for improvement of electrical conductivity of electrically conductive adhesives with isotropical electrical conductivity. Conductivity level depends, among other things, on level of aggregation of filler particles. The research has been focused on improvement of electrical properties by better aggregation of filler particles in adhesive. Following methods have been tested with the goal to support aggregation: ultrasound mixing for $60 \mathrm{~s}$, rotary mixing for $60 \mathrm{~s}$, and addition of a small amount of $\mathrm{AgNO}_{3}$. The goal of addition of this salt has been to decrease Coulomb forces between particles of filler. Four types of formulations have been used for experiments. One-component formulations: adhesives bisphenol epoxy resin with $75 \%$ (wt.) silver flakes, bisphenol epoxy resin with $75 \%$ (wt.) silver flakes + $10 \%$ (wt.) silver nanoparticles, bisphenol epoxy resin with $65 \%$ (wt.) silver flakes $+20 \%$ (wt.) silver nanoparticles. Two component formulation: bisphenol epoxy resin with $55 \%$ (wt.) silver flakes. It has been found that ultrasound mixing has low influence only to the value of the electrical conductivity of adhesive, rotary mixing before application of the adhesive improves electrical conductivity significantly; addition of $\mathrm{AgNO}_{3}$ into the adhesive has improved electrical conductivity of two formulations and has had almost no effect for two other formulations. As a part of this work the course of electrical conductivity of adhesives during the curing time has also been investigated.
\end{abstract}

\section{Introduction}

There are two basic types of electrically conductive adhesives: adhesives with isotropical and adhesives with anisotropical electrical conductivity. This paper is focused on adhesives with isotropical electrical conductivity.

Electrical as well as mechanical properties of adhesives with isotropical electrical conductivity are worst in comparison with the properties of solders in general. Therefore there are tested different methods for improvement of these properties. One group of them is based on addition of particles of different types into adhesives [1]; another group is focused at processing adhesives before application. We have tested methods of both these groups.

Improvement of properties of conductive adhesives is a difficult problem. One possible solution is to use, instead of a standard type of filler (silver flakes for isotropically conductive adhesives), carbon nanotubes or silver coated carbon nanotubes. Carbon nanotubes improve mechanical parameters of adhesive, if they are silver coated; electrical properties are improved, too [2]. The main disadvantage of such the solution is extremely high price. This fact limits this technology.

Value of electrical conductivity of electrically conductive adhesives is approximately five or more times lower in comparison with the electrical conductivity of solders. The reason of worst electrical conductivity is the structure of a conductive net in adhesive. Quality of this net depends on quality of contacts among conductive particles of filler. The total resistance of this net is composed of the resistance of filler particles and of the resistance of contacts among them. Therefore the electrical conductivity of electrically conductive adhesive has to be lower in comparison with the electrical conductivity of solder in principle, if the same material of filler like material of solder is used.

It is in evidence that the total electrical conductivity of electrically conductive adhesive depends on quality and density of a conductive net created inside the adhesive by filler particles. Parameters of conductive net depend, instead other things, on a level of aggregation of filler particles. The goal of this work has been to find a method, which would intensify aggregation and this way improve electrical conductivity of adhesives.

\section{Theoretical background}

There are three components of electrical conductivity of electrically conductive adhesives: conductivity of filler particles, tunnel conductivity of contacts between filler particles and constriction conductivity of contacts between filler particles.

As for the conductivity of filler particles, this conductivity is high, because such metals as silver, gold, palladium or nickel are used for their fabrication. Worst electrical conductivity have particles of plastic materials covered with a thin metal film, which are used in some types of adhesives with anisotropic electrical conductivity.

Tunnel conductivity is joined with the fact that electron has also wave properties. Therefore it is able to get over a potential barrier, which is higher than its energy.

Constriction conductivity is joined with a small contact between particles, mostly between spherical 
particles. If the current passes through such a contact, the current lines are compressed into a very small area, the current density in this area is very high and therefore the total conductivity of such the arrangement decreases.

The electrical conductivity of electrically conductive adhesives depends strongly on the dimensions of filler particles. Adhesives with filler consisting of conductive balls or flakes have the percolation threshold in limits from $50 \%$ (wt) to $70 \%$ (wt) usually.

Decrease of this concentration causes overrun of the percolation threshold and dramatic decrease of the conductivity of adhesive. If concentration of conductive particles in adhesive overruns the percolation threshold and follows up increase, the conductivity of adhesive increases too, but the increase is substantially smaller in comparison with the increase of the electrical conductivity in area of the percolation threshold.

When filler of adhesive consists of a mixture of micro and nanoparticles, the percolation threshold occurs at lower concentrations of filler particles usually that it would occur when filler would consist of microparticles only.

Considering dimensions of nanoparticles, their number is substantially higher than number of microparticles if the same concentration (wt) of these particles is in adhesive. If they are properly distributed, they can create, together with the microparticles, a very dense conductive net. However, the number of contacts between nanoparticles and microparticles and between nanoparticles mutually in such a net is higher than number of contacts between microparticles only, when the adhesive is filled with this filler. Also the area of contacts between microparticles is higher than the area of contacts between nanoparticles. This is a reason why adhesives filled with nanoparticles or adhesives filled with a mixture of micro and nanoparticles have lower electrical conductivity in comparison with adhesives filled with microparticles only.

There are following forces in electrically conductive adhesive, which influence aggregation of filler particles: small attractive Van der Waals forces, which act on small distance only (approx. $10 \mathrm{~nm}$ ), repulsive Coulomb forces and forces of the resistance of a medium (resin), joined with its viscosity. Coulomb forces and forces of the resistance of a medium counteract aggregation of filler particles. These forces form energetic barriers between particles.

This energetic barrier can be surpassed by a particle by supply of external energy. This energy can be thermal energy, or another type of energy, which will decrease level of this energetic barrier. It has been tested if decrease of this barrier between particles can be reduced by ultrasonic or rotary mixing.

\section{Experimental}

Four types of formulations have been used for testing. One-component adhesive with bisphenol epoxy resin filled with silver flakes ( $75 \%$ wt.), the same adhesive with addition of $10 \%$ silver nanoparticles. The one- component bisphenol epoxy adhesive with a mixture of $65 \%$ (wt.) silver flakes and $20 \%$ (wt.) silver nanoparticles. The composition of two-component adhesive has been bisphenol epoxy resin with $55 \%$ (wt.) silver flakes. Silver nanoparticles have been grains with dimensions between 3 to $55 \mathrm{~nm}$.

One way under test how to improve the electrical conductivity of adhesive has also been to add $\mathrm{AgNO}_{3}$ into the adhesive. The idea has been as follows: in adhesive are, before curing, filler particles surrounded by molecules of polar solvent (initiator). $\mathrm{AgNO}_{3}$ added into the adhesive will decay in positive ions $\mathrm{Ag}^{+}$and negative ions $\mathrm{NO}_{3}{ }^{-}$due to the curing temperature. $\mathrm{Ag}^{+}$ions will attract negative ions of initiator surrounding filler particles; this way will decrease of Coulomb forces between them and support their aggregation.

For testing of influence of $\mathrm{AgNO}_{3}$ on properties of adhesives two types of formulation have been prepared: formulations without $\mathrm{AgNO}_{3}$ and formulations with small addition of this salt (concentration has been $1,2 * 10^{-5}$ $\mathrm{mol} / \mathrm{g}$ ). After addition of $\mathrm{AgNO}_{3}$ adhesive has been mixed using an ultrasound mixer for $30 \mathrm{~s}$.

Next process, which has been tested for improvement of electrical conductivity of adhesives, has been a process of mixing. Two types of mixing have been used: ultrasound mixing and rotary mixing.

Ultrasound mixing has been carried out in an ultrasound mixer Labora UVM at the frequency of $40 \mathrm{kHz}$ for $60 \mathrm{~s}$. Rotary mixing has been carried out using a rotary mixer Bionic. The mixing conditions have been 13000 turns/min, a conical mixing tool with diameter of 2,5 $\mathrm{mm}$ has been used.

Specimens have been formed as pastilles of adhesive with cast-in electrodes making the four-point electrical resistivity measurement possible. The diameter of the pastilles has been $12 \mathrm{~mm}$ and their height $3 \mathrm{~mm}$. The specimen is schematically shown in Fig. 1. Electrodes have been gold wires with the diameter of $0.5 \mathrm{~mm}$.

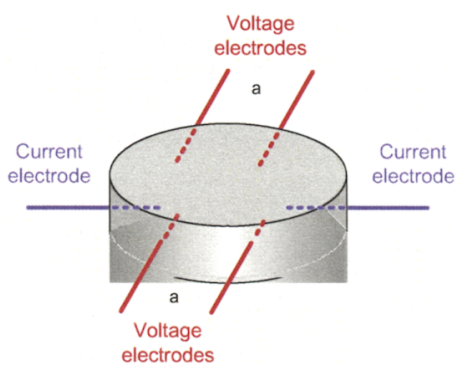

Fig. 1 Specimen for measurement of electrical resistivity of electrically conductive adhesives, $a=3 \mathrm{~mm}$

Resistivity of adhesive can be calculated using a formula:

$$
\rho=\frac{U}{I} b K_{s} K_{r} K_{t}
$$


Where:

$U$... voltage measured between the opposite voltage electrodes.

$I$... current flowing between the current electrodes.

$b \ldots$ thickness of a specimen.

$K_{s} \ldots$ correction factor dependent on the diameter of the measured specimen and on the distance of the voltage electrodes. It has been calculated that $K_{s}=2,9289$.

$K_{r} \quad$... correction factor dependent on the thickness of the specimen and on the distance of the voltage electrodes. It has been calculated that $K_{r}=0,9214$.

$K_{t} \quad \ldots$ correction for the temperature. This factor is calculated using the formula:

$K_{t}=1+0,01(t+24)$

Where:

t ... temperature $\left({ }^{0} \mathrm{C}\right)$

The conductivity has been calculated using the formula:

$$
\sigma=\rho^{-1}
$$

The four-point measurement of the resistivity has been carried out using two current electrodes and two pairs of the voltage electrodes. The voltage has been measured between opposite electrodes. Two pairs of the voltage electrodes have been used to verify homogeneity of the measurement. The measuring current $25 \mathrm{~mA}$ has been used for the measurement. The fixture used for the measurement is shown in Fig. 2.

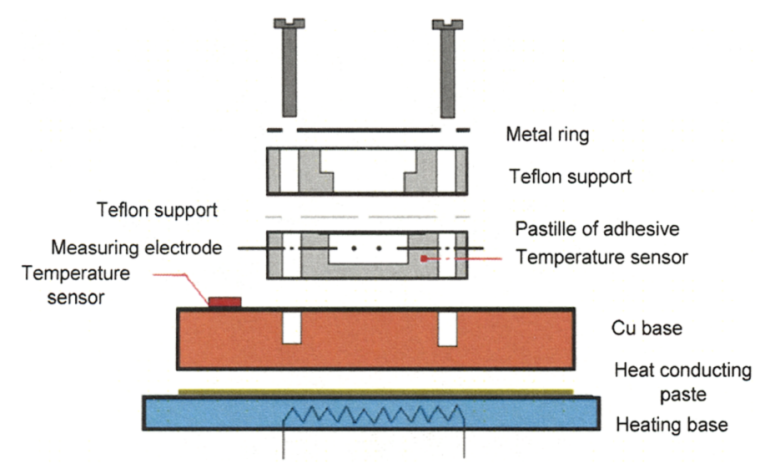

Fig. 2 Fixture used for curing of adhesive and measurement of resistivity during curing

The current source has been a sinus generator Tesla SG 8 completed with a band pass filter. The measuring frequency has been $19217 \mathrm{kHz}$. The current flowing through the specimen has been measured using a resistor $200 \Omega$ connected in series with the current electrodes. The voltage drop on this resistor has been measured using a voltmeter HP 4284A. The voltage between the voltage electrodes has been measured with a lock-in amplifier Stanford Research Systems SR 830.
Heating base with stabilization and control of the temperature, on which the specimen has been located, has been of the type Yellow Line MST.

\section{Results and Discussion}

There are two processes, which occur during curing of electrically conductive adhesive: polymerization of the binder and aggregation of particles of the filler. Polymerization of the binder is a complicated chemical process; the time of polymerization is usually between 20 to $40 \mathrm{~min}$. When the polymerization has finished, the heating has been switched out, the temperature of the specimen has decreased and the conductivity of adhesive has increased. This fact is connected with additional polymerization of the binder and with the decrease of the volume of adhesive (the total volume decrease is approx. $7 \%$ in dependence on the type of resin, which has been used [3]).

The mechanism of polymerization and aggregation, when $\mathrm{AgNO}_{3}$ is added into the adhesive, is as follows: solvent (initiator of polymerization) is partially inbuilt and partially vaporized by degrees during curing of the adhesive. Molecules of the solvent are ripped off the surface of filler particles and the surface charge of these particles decreases. Therefore probability that these particles will aggregate increases. The ions of $\mathrm{Ag}^{+}$can, due to thermal movement, fill gaps between particles of the filler or can aggregate mutually and clusters of these particles can fill gaps between filler particles and to increase conductivity of adhesive.

Influence of different methods for support of aggregation of filler particles in adhesives on conductivity of adhesives are show in Fig. 3 to Fig. 7.

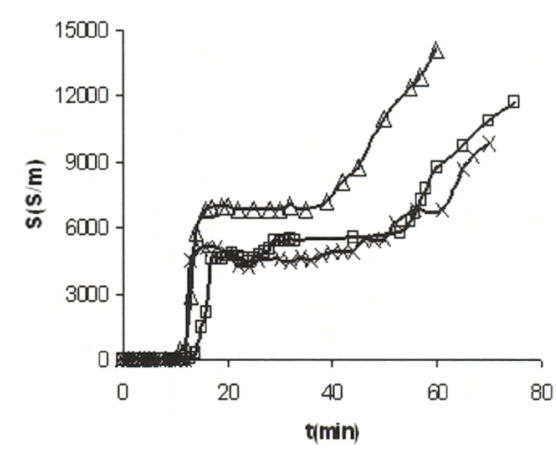

Fig. 3 Conductivity of different formulations in dependence on the curing time without support of aggregation. $\Delta \ldots$ basic adhesive, $\square \ldots$ basic adhesive $+10 \%$ nano, $x$... basic adhesive $+20 \%$ nano, 0 ... two-component adhesive 


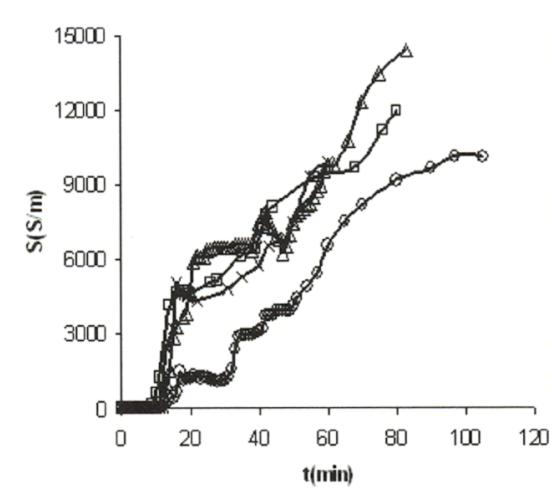

Fig. 4 Conductivity of different formulations in dependence on the curing time. Aggregation has been supported by ultrasound mixing before application of adhesives. $\triangle \ldots$ basic adhesive, $\square$... basic adhesive $+10 \%$ nano, $\mathrm{x}$... basic adhesive $+20 \%$ nano, 0 ... two-component adhesive

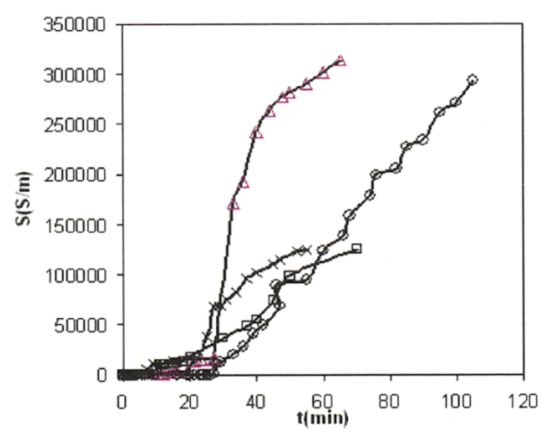

Fig. 5 Conductivity of different formulations in dependence on the curing time. Aggregation has been supported by rotary mixing before application of adhesives.

$\Delta \ldots$ basic adhesive, $\square . .$. basic adhesive $+10 \%$ nano,

$x \ldots$ basic adhesive $+20 \%$ nano, 0 ... two-component adhesive

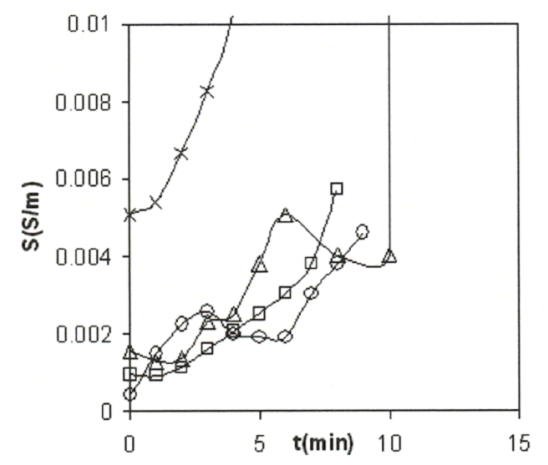

Fig. 6 Conductivity of basic adhesive during starting phase of curing.

$\Delta \ldots$ no support of aggregation, $\square \ldots \mathrm{AgNO}_{3}$ added,

$x$... rotary mixing before application, $\mathrm{o}$... ultrasound mixing before application

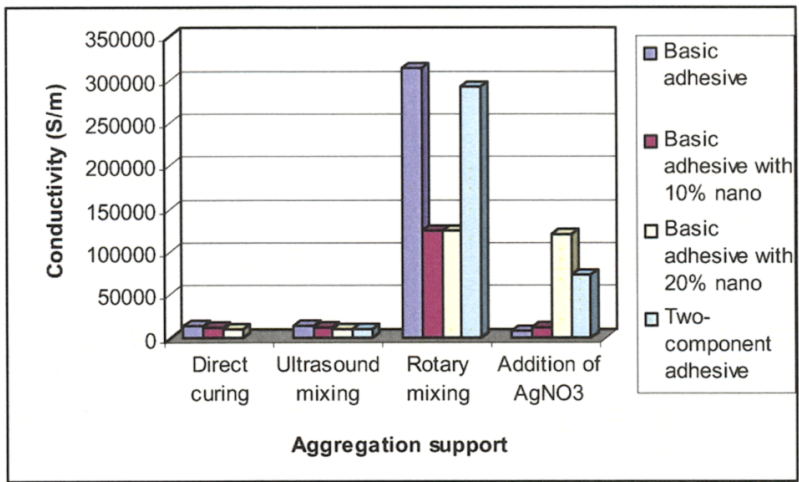

Fig. 7 Comparison of conductivity of tested formulations in dependence on aggregation support

\section{Conclusions}

Electrical conductivity of adhesives, which have not been mixed before application, has been lower than after mixing. The reason is sedimentation of filler particles.

Ultrasound mixing is not too effective. The reason is that the particles are homogeneously dispersed in the volume, among them are too big distances and aggregation is more difficult.

Rotary mixing is a very effective technology for improvement of electrical conductivity of adhesives.

The reason is that during rotary mixing arise shear forces, which lower energetic barriers between particles and contribute to their aggregation.

Addition of $\mathrm{AgNO}_{3}$ into the adhesives has had a positive effect to electrical conductivity for two formulations only. However, this technology seems to be promising and it will be deeper investigated.

It has been found that the most effective technique for support of aggregation and improvement of electrical conductivity of electrically conductive adhesives is the rotary mixing before application of adhesive.

\section{Acknowledgments}

The work has been carried out as a part of a project "Diagnostics of Materials", number MSM6840770021

\section{References}

1. Lee, H. H., Chou, K. S., Shih Z. W.: "Effect of nanosized silver particles on the resistivity of polymeric conductive adhesives." International Journal of Adhesion and Adhesives, Vol. 25, Issue 5 (2005), pp. 437-441

2. Wu, H.P., Wu, X.J., Ge, M.Y., Zhang, G.Q., Wang, Y.W., Jiang, J.: Properties investigation on isotropical conductive adhesives filled with silver coated carbon nanotubes. Composites Science and Technology: Volume 67, Issue 6, May 2007. pp. 1182-1186.

3. Su, B.: Electrical, Thermomechanical and Reliability Modeling of Electrically Conductive Adhesives. Dissertation. Georgia Institute of Technology, May 2006, pp. 49-52. 